\title{
Lipid Accumulation Product (LAP) and Waist Circumference to Height Ratio (WHtR) Associated with Free Androgen Index but not with serum Testosterone and Androstendione in Women with PCOS
}

\author{
Kuliczkowska-Plaksej J ${ }^{1}$, Milewicz $\mathrm{A}^{1}$, Laczmanski $\mathrm{L}^{1}$, Jedrzejuk $\mathrm{D}^{1}$, Lwow $\mathrm{F}^{2}$, Bolanowski $\mathrm{M}^{1}$ \\ 1 Department of Endocrinology, Diabetology and Isotope Therapy, Wroclaw Medical University, Poland \\ 2 Department of Health Promotion, Faculty of Physiotherapy, University School of Physical Education, Wroclaw, Poland
}

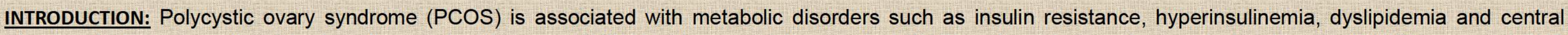

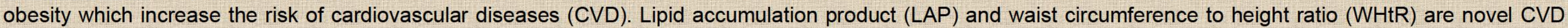
risk predictors based on the assessment of waist circumference, height and serum triglycerides.

AIM OF THE STUDY: The aim of our study was to evaluate the LAP and WHtR associations with hyperandrogenemia in women with PCOS.

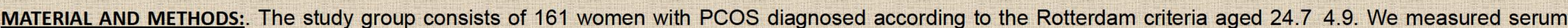

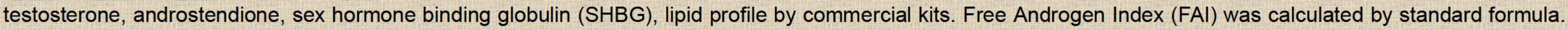

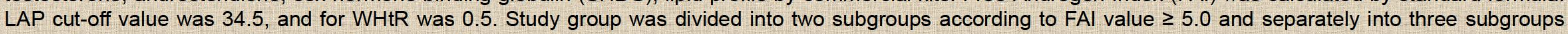
according to serum testosterone and androstendione concentrations.

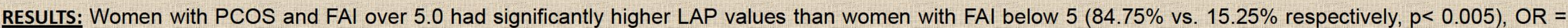

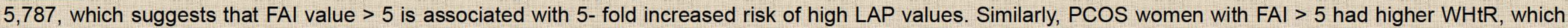

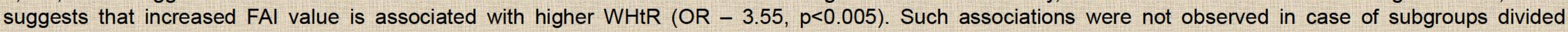

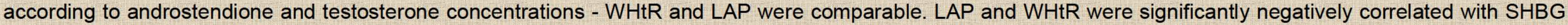
concentration and positively with FAl values, but not with serum testosterone and androstendione levels.

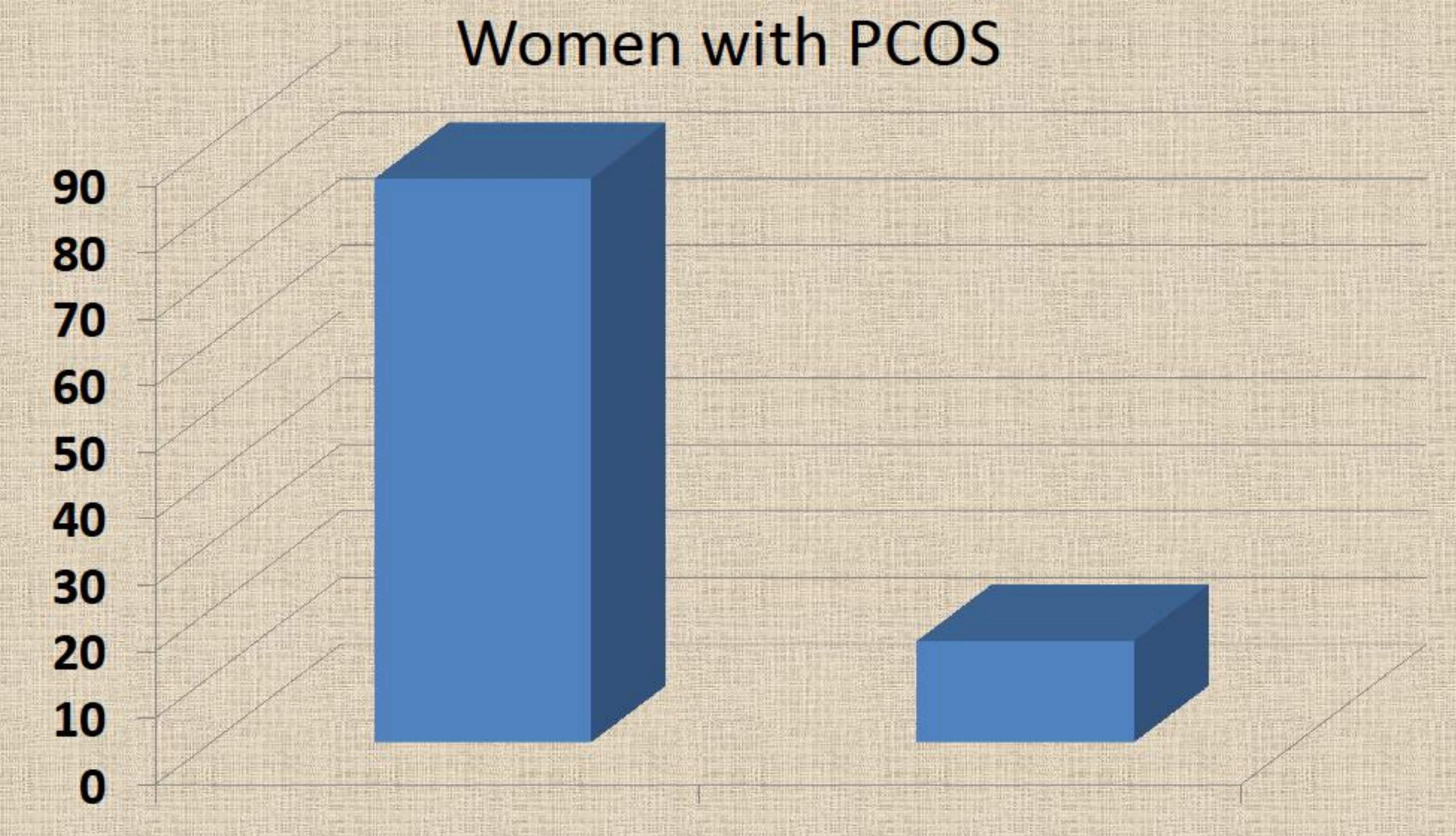

FAI $>5$

FAI $<5$
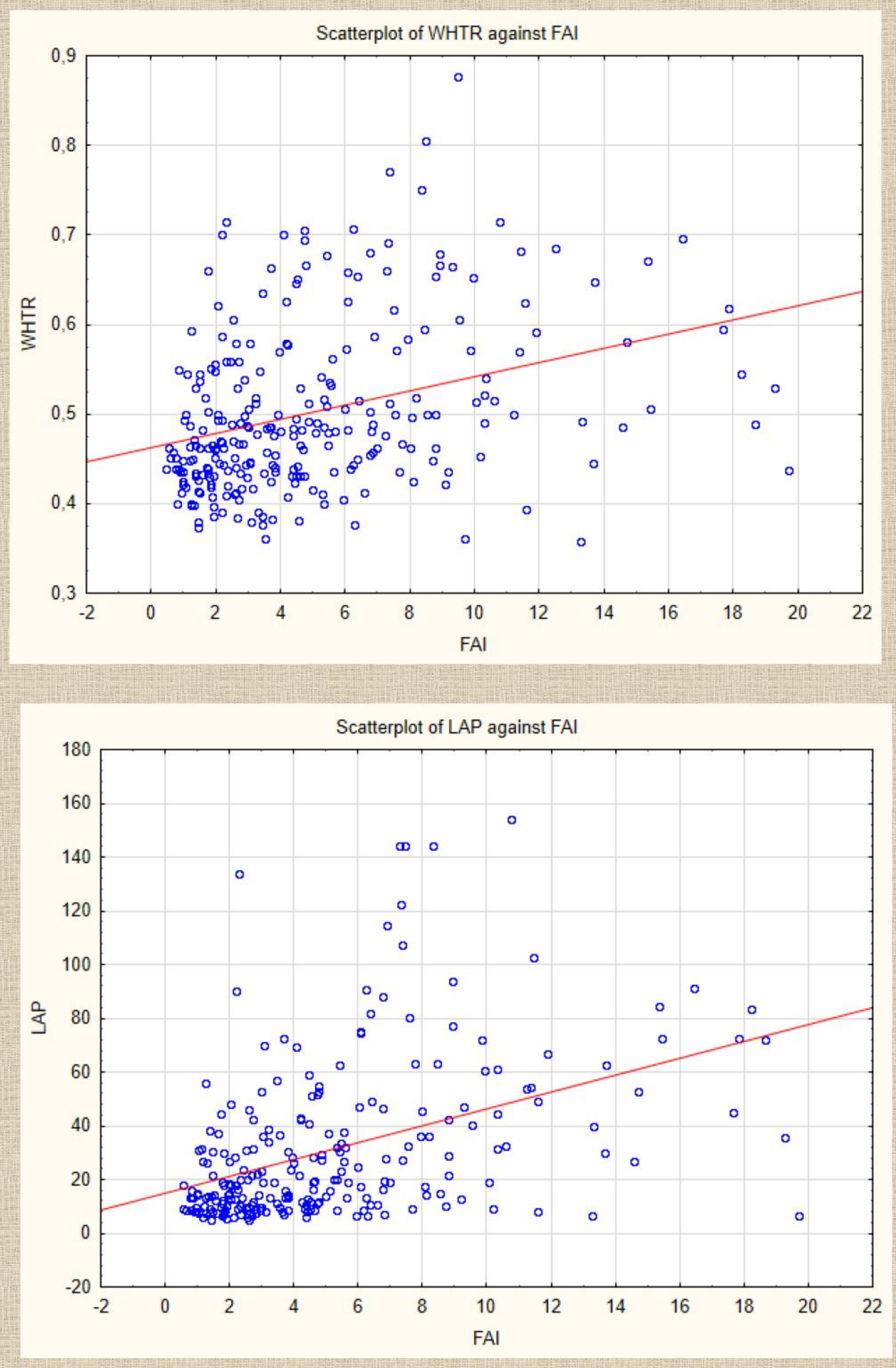
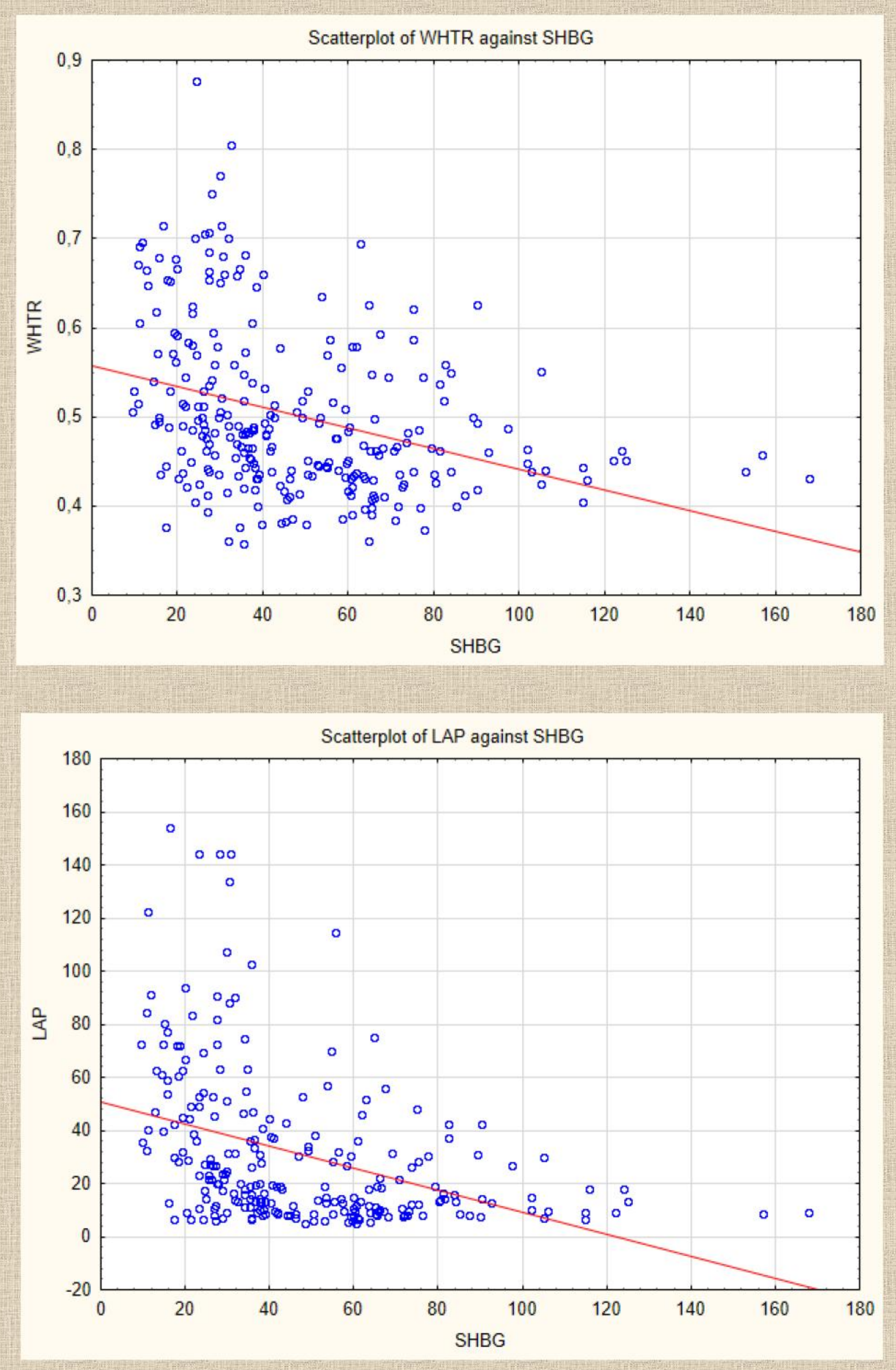

\section{CONCLUSION:}

Hyperandrogenemia expressed as $\mathrm{FAl} \geq 5.0$ is associated with significantly higher values of novel CVD risk predictors - LAP and WHIR in women with PCOS. Serum SHBG level as well as FAl value are good predictors of CVD risk in women with PCOS. 